\title{
Sistema móvil de teleasistencia médica para la atención en tiempo real de casos de urgencia
}

\section{Mobile Telecare System for Real-Time Medical Emergency Care}

\author{
Rivera-Rodríguez R. \\ Facultad de Ingeniería Campus Mexicali \\ Universidad Autónoma de Baja California \\ Baja California Norte, México \\ Correo:rrivera@cicese.mx \\ Serrano-Santoyo A. \\ Dirección de Impulso a la Innovación y Desarrollo \\ Centro de Investigación Cientifica y de \\ Educación Superior de Ensenada, \\ Ensenada, Baja California, México \\ Correo:serrano@cicese.mx
}

\author{
Tamayo-Fernández R. \\ Facultad de Ingeniería Campus Mexicali \\ Universidad Autónoma de Baja California \\ Baja California Norte, México \\ Correo:rtamayo@cicese.mx \\ Armenta-Ramade A. \\ Dirección de Impulso a la Innovación y Desarrollo \\ Centro de Investigación Cientifica y de \\ Educación Superior de Ensenada, \\ Ensenada, Baja California, México \\ Correo:aarmenta@cicese.mx
}

Información del artículo: recibido: mayo de 2007, reevaluado: mayo de 2008, aceptado: febrero de 2011

\section{Resumen}

Este artículo describe un sistema móvil de teleasistencia médica para apoyar la atención de casos de emergencia, utilizando servicios de transmisión de signos vitales y videoconferencia en tiempo real. Así, en forma remota, los médicos especialistas pueden dar instrucciones precisas y oportunas a los paramédicos, con el propósito de mejorar la atención médica que recibe el paciente. Se analizan los aspectos técnicos de conectividad y factibilidad para la puesta en operación del sistema desde el punto de vista de cobertura inalámbrica, normatividad y tecnología actualmente disponibles en México, en donde los resultados obtenidos indican que la elección del servicio de comunicación inalámbrica es importante para soportar este servicio. Finalmente, se presentan resultados de la evaluación con diferentes tecnologías de comunicación inalámbrica y los retos e implicaciones en la adopción tecnológica por parte de los actores en la sala de emergencia y la unidad médica móvil, para que el sistema propuesto sea utilizado de manera eficiente en el entorno hospitalario.

\section{Descriptores}

- telesalud

- comunicaciones inalámbricas

- 3G

- videoconferencia SIP

- UML

- emergencias médicas 


\begin{abstract}
This article describes a mobile medical telecare system installed in moving vehicles to support emergency cases, it provides videoconference and vital signs transmission services in real time. Thus remote specialist doctors can give accurate and timely instructions to the paramedics, saving time and improving patient care process. We present an analysis of the technical issues involving connectivity and feasibility to operate the system in a wireless environment considering the regulatory aspects currently existing in Mexico, where the results obtained indicate that the choice of wireless communication service is important to support this service. We present the evaluation results with different wireless communication technologies, and the challenges and implications of the adoption of technology are also discussed considering the elements involved in a hospital environment.
\end{abstract}
Keywords
- e-health
- wireless communications
- 36
- videoconference
- UML
- medical emergencies

\section{Introducción}

La teleasistencia médica puede considerarse como una actividad en la cual se ofrece atención médica a distancia, en donde las tecnologías de la información y comunicaciones (TIC), en particular, los sistemas de comunicaciones inalámbricas de tercera generación (3G) y de espectro disperso (como WiFi y WiMAX) (Li et al., 2007), proveen mayor capacidad de transmisión de datos, beneficiando el envío y recepción de información médica como: signos vitales, expedientes clínicos, imágenes médicas, y videoconferencia. En este trabajo, se estudia el escenario de la teleasistencia médica en movimiento aplicada a casos de emergencia, traslados delicados o especializados. En este caso, personal especializado en la sala de urgencias del hospital o centro de control, monitorea y analiza la información transmitida desde una unidad médica en movimiento, lo que le permite emitir órdenes de acción al paramédico en la ambulancia durante el traslado del sitio de la emergencia hacia el hospital. Un sistema de esta naturaleza puede utilizarse en traslados que requieran monitoreo continuo e involucren aspectos de seguridad y protección civil.

Una característica importante del sistema desarrollado es la aplicación de la videoconferencia en movimiento, en donde los aspectos de integridad, restablecimiento de las señales, permanencia de la sesión de trabajo, así como la cobertura inalámbrica y las capacidades de ancho de banda del canal son fundamentales para lograr un sistema robusto y acorde a las necesidades de un entorno prehospitalario.

\section{Trabajo relacionado}

En México se han realizado varios esfuerzos en el ámbito de la telemedicina, uno de ellos es el caso del Institu- to de Seguridad y Servicios Sociales de los Trabajadores del Estado (ISSSTE), el cual contempla la consulta médica a distancia utilizando enlaces satelitales y líneas dedicadas para el acceso a Internet (Gómez, 2001). Otros esfuerzos son los del Instituto Mexicano del Seguro Social (IMSS), que se han enfocado a sistemas para la implantación de infraestructura en hospitales digitales. Los proyectos del IMSS se han enfocado en la aplicación del expediente clínico electrónico (ECE) utilizando tecnologías para el despliegue de la información, movilidad de médicos y pacientes, entre otras, (Hospitales Digitales, 2007).

Existen otros trabajos de investigación en el área de la telesalud en diferentes instancias académicas y hospitalarias. A la fecha, se han identificado los retos y beneficios que se tienen al implantar y operar una red de comunicaciones inalámbrica en un hospital (Cypher et al., 2006) para proveer un ambiente de conectividad ubicua.

Por otro lado, en Istepanian et al. (2004) se describen los conceptos de la llamada disciplina de "salud móvil" (M-Health), en la cual se define el uso de las tecnologías inalámbricas en forma general, considerando el caso de movilidad en la provisión de servicios y cuidados médicos. Existen otros trabajos (Poon et al., 2006) que se enfocan en la aplicación de redes de sensores y métodos biométricos al ofrecer servicios de salud móvil.

En los casos anteriores, los esfuerzos se concentran en resolver problemas de movilidad o seguridad utilizando el monitoreo de signos vitales. Sin embargo, ninguno de los trabajos antes mencionados aborda la aplicación de la videoconferencia en movimiento para casos de urgencias en ambientes prehospitalarios.

En este trabajo se considera importante la videoconferencia, ya que es una herramienta de apoyo para la inspección visual del paciente por parte de un especialista ubicado en un hospital. El hecho de que el especia- 
lista pueda tener una imagen de la condición clínica del paciente (es decir, que pueda ver la herida, pueda ver la reacción del paciente a un estímulo, etc.) le ayuda a tener un mejor diagnóstico y por ende, dar instrucciones más acertadas para la atención del paciente en su traslado hacia el hospital.

\section{Descripción del sistema}

El sistema de teleasistencia médica en movimiento (SITAMM), permite la transmisión en tiempo real y en movimiento de videoconferencia y signos vitales desde una unidad móvil de emergencia (UME), la cual puede ser una ambulancia o cualquier otra unidad que requiere de traslados con monitoreo continuo. El sistema considera en su diseño las condiciones adversas del medio inalámbrico y las características limitadas de la capacidad en ancho de banda del canal. La figura 1 presenta el escenario completo de comunicación.

Como se puede observar en la figura 1, el SITAMM está compuesto por cuatro elementos clave:

1) el SM-SITAMM ubicado dentro de una UME,

2) el SC-SITAMM ubicado dentro de las instalaciones de un hospital,

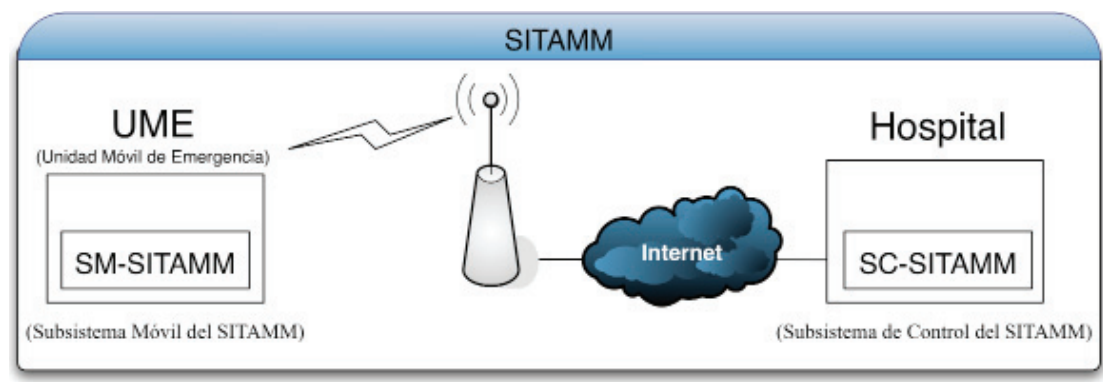

Figura 1. Elementos del SITAMM

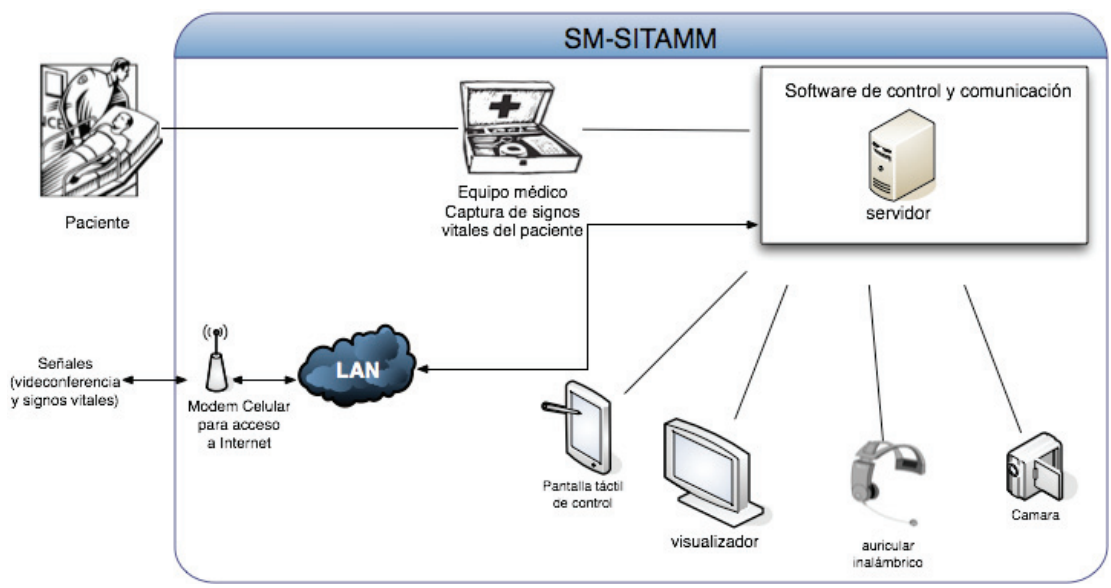

Figura 2. Subsistema móvil del SITAMM
3) un canal inalámbrico celular, $y$

4) un acceso a Internet que permite la comunicación de datos a la red del hospital.

\section{a) Subsistema móvil del SITAMM (SM-SITAMM)}

La figura 2 muestra los elementos del SM-SITAMM, y se observa que tiene acceso a la red de datos del hospital a través de un canal inalámbrico celular, el cual es monitoreado constantemente para determinar su afectación por condiciones adversas al canal, como desvanecimientos y multitrayectorias (Rappaport, 2002), afectando la pérdida de información. El objetivo es ajustar dinámicamente los parámetros de transmisión y mantener la sesión de videoconferencia activa, de tal forma que las condiciones del canal afecten lo menos posible la transmisión de información.

La adquisición de los signos vitales del paciente por medio del SM-SITAMM, requiere de un equipo médico digital conectado a un servidor, donde se encuentra instalado el software para la captura de datos del monitor de signos vitales, control de sesión de videoconferencia y captura de señales digitales de los dispositivos de audio y video (auricular inalámbrico y cámara digital). Para no interferir en las funciones propias del paramédico (atención del paciente), el sistema provee una interfaz de control que puede activarse antes de arribar al lugar del siniestro. Esta interfaz utiliza una pantalla táctil para manipular de manera sencilla (con un solo botón) el inicio y fin de la videoconferencia, así como el envío de signos vitales al hospital. El sistema cuenta también con un monitor LCD de 20 pulgadas para el despliegue de la información y la videoconferencia.

El sistema de videoconferencia fue desarrollado utilizando el protocolo de inicialización de sesión (SIP, Session Initialization Protocol) aprovechando su flexibilidad y versatilidad (RFC 3261, 2002). El protocolo SIP fue programado para enviar la información de signos vitales dentro del canal de señalización de la videoconferencia, facilitando los procesos de gestión en el sistema. La señal de videoconferencia y signos vitales son transmitidos a través de la red celular hacia el subsistema de control del SITAMM (SC-SITAMM). 
b) Subsistema de control del SITAMM (SC-SITAMM)

La figura 3 muestra los elementos del SC-SITAMM, el cual consta de un acceso a Internet para la comunicación con la unidad móvil de emergencia, un servidor para la señalización y registro de clientes SIP de cada UME, y otro servidor con el software para el manejo del sistema de videoconferencia y recepción de signos vitales de las diferentes UMEs. Al igual que su contraparte

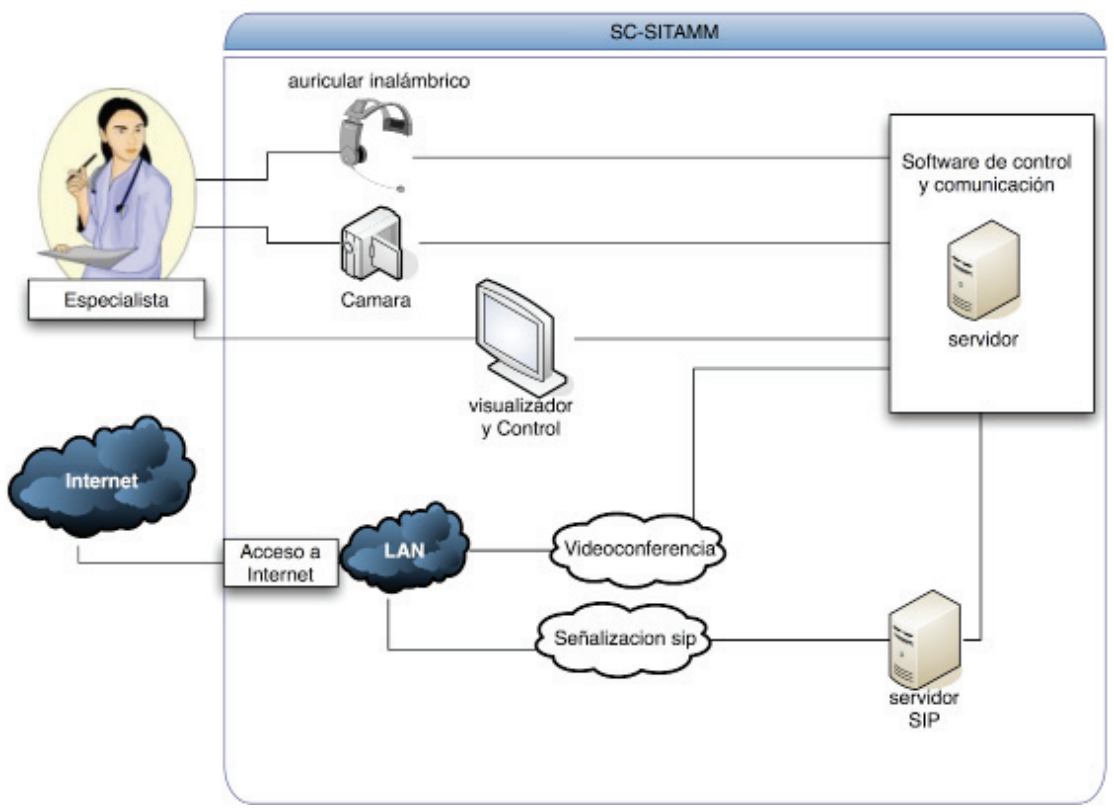

Figura 3. Subsistema de Control del SITAMM (SC-SITAMM)

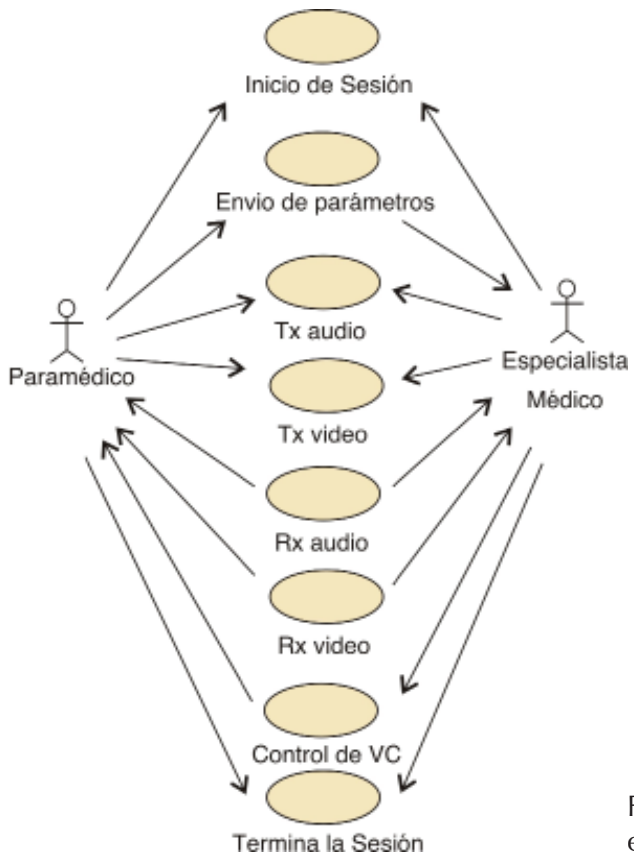

Figura 4. Caso de uso para el establecimiento de videoconferencia móvil, el SC-SITAMM utiliza un auricular inalámbrico, una cámara digital y un pizarrón electrónico digital táctil para las funciones de panel de control, visualización de signos vitales y videoconferencia.

El SC-SITAMM recibe la información de videoconferencia y signos vitales a través de Internet. Antes de desplegar la videoconferencia, los datos de signos vitales son extraídos utilizado mensajes de la señal de señalización del protocolo SIP para posteriormente ser enviados al sistema de visualización en el hospital. La información recibida en el sistema de visualización es analizada por un especialista médico, quien da instrucciones al paramédico que atiende al paciente en la UME sobre los procedimientos a seguir relacionados con el estado del paciente. El sistema de videoconferencia funciona como una herramienta de apoyo para los médicos especialistas, quienes a distancia pueden utilizar este medio para dar instrucciones precisas y oportunas a los paramédicos, y así poder reducir la ventana terapéutica que recibirá el paciente. Se realiza un diagnóstico prehospitalario sustentado en actividades de hallazgos físicos por inspección visual, así como preguntas para determinar elementos de reacción en el paciente.

El uso de este sistema extiende las capacidades del hospital al lugar de la emergencia mejorando procesos de recepción del paciente, optimizando la programación de estudios, salas de operación y medicamentos necesarios dependiendo del caso de emergencia.

\section{Funcionamiento del SITAMM}

La sesión de videoconferencia es iniciada por el paramédico presionando un ícono sobre la pantalla táctil de la UME. El programa de control y comunicaciones desarrollado se encarga de establecer la sesión de videoconferencia de forma automática permitiendo al paramédico enfocarse en atender al paciente. 
La figura 4 muestra el modelo UML (Unified Modeling Language) del 'caso de uso' (Bennett et al., 2001) de la sesión de videoconferencia entre dos actores. El presente artículo considera a los actores como el paramédico y el especialista.

En la aplicación desarrollada se incluye el 'caso de uso' denotado como "envío de parámetros", en el que periódicamente se envía información de las condiciones del canal de comunicación y la cual se utiliza para adaptar los parámetros de calidad de la videoconferencia.<smiles>CC(C)C(C)C</smiles>

Paciente

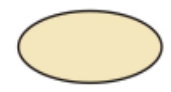

Captura de signos vitales
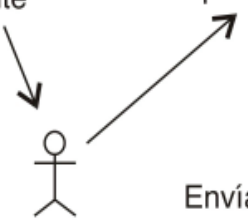

\section{E}
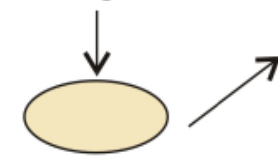
Señalización SIP Señalización SIP vía los signos vitales

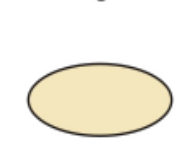

Validación de datos de Signos vitales
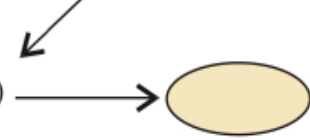

Envia datos al

SC-SITAMM
Este 'caso de uso' tiene el objetivo de mantener la sesión, o restablecerla automáticamente cuando se presente desvanecimiento de potencia, congestión de la red celular o de Internet, o haya un 'handoff' (proceso en el que la terminal móvil cambia de una celda de la red celular a otra porque ofrece mejores prestaciones en la calidad de la comunicación).

La figura 5 presenta el 'caso de uso' denotado como "envío de signos vitales", en el cual se adquieren los signos vitales del paciente para su envío utilizando el canal de señalización SIP. Los signos vitales encapsulados son recibidos, extraídos y desplegados en el SC-SITAMM localizado en el hospital.

\section{Resultados y discusión}

La figura 6 muestra el SM-SITAMM instalado en una UME experimental, en ésta se aprecia la imagen de videoconferencia (lado izquierdo), el monitor de signos vitales (lado derecho, abajo) y el panel de control sensible al tacto (lado derecho, arriba). La figura 7 muestra un ejemplo de la interfaz de usuario del SC-SITAMM, el cual se encuentra en las instalaciones del hospital.

Los valores mostrados en la figura 7 no representan un caso real de emergencia, su propósito es mostrar que la interfaz presenta diferentes áreas de información, con las cuales el médico puede conocer los signos vitales del paciente y utilizar la videoconferencia para realizar hallazgos físicos y conocer más sobre la condición del paciente.

a) Tecnologías inalámbricas utilizadas

El sistema desarrollado hace uso de tecnologías inalámbricas de segunda generación (2.5G) y tercera generación (3G). El sistema fue probado en redes tipo GSM (Global System for Mobile Communications) y CDMA (Code Division Multiple Access), las cuales utilizan los esquemas de comunicación de datos EDGE (Enhanced Data Rates for GSM Evolution)

Figura 6. Interior del SM-SITAMM 


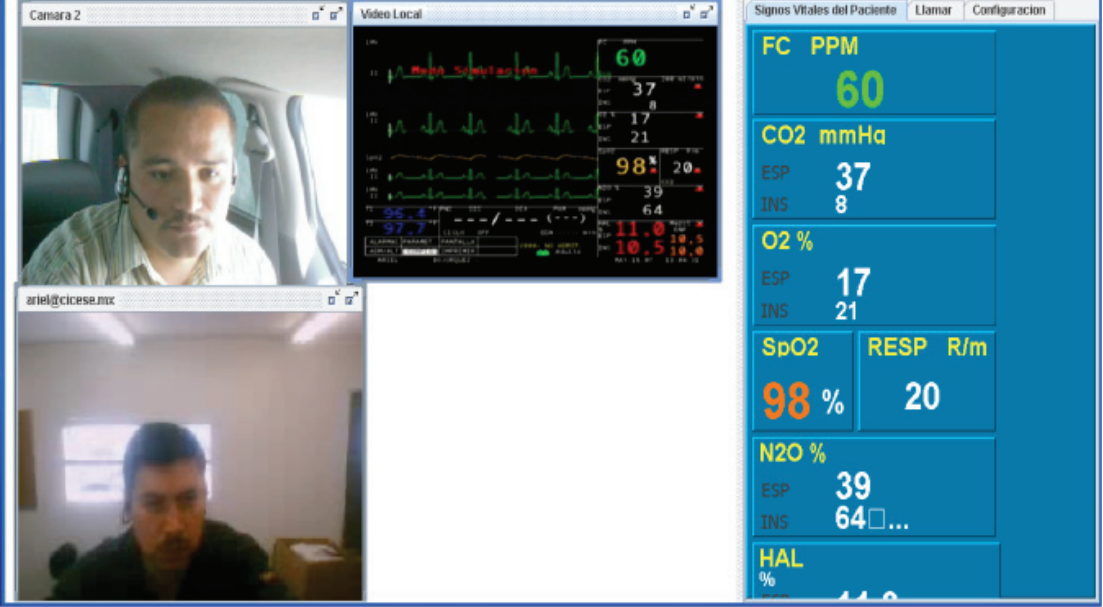

Figura 7. Interfaz de usuario del SCSITAMM
(Ericsson, 2007) y Ev-DO (Evolution Data Optimized), (Qi et al., 2003), respectivamente, como se describe a continuación.

El esquema de comunicación EDGE es un estándar que utiliza técnicas de modulación y codificación avanzadas, diseñado para incrementar la velocidad de datos y la capacidad de la red en sistemas GSM. EDGE permite tasas de datos de hasta $48 \mathrm{~Kb}$ ps por canal y es posible incrementarla hasta $384 \mathrm{Kbps}$ agregando varios canales en una misma sesión. Sin embargo, resultados recientes (Ericsson, 2007), indican que en la práctica no se superan los $200 \mathrm{Kbps}$ promedio y conforme el usuario se desplaza a mayor velocidad, la tasa de transmisión se reduce considerablemente.

Por otro lado, Ev-DO es un estándar para transmisión de datos a alta velocidad basado en CDMA, que permite velocidades de hasta $2.4 \mathrm{Mbps}$ en su versión original (revisión 0) y de hasta 3.1 Mbps en la modificación A (revisión A). Actualmente, en algunos países se encuentra disponible la modificación B (revisión B) que permite tasas cercanas a los $5 \mathrm{Mbps}$. Al igual que en EDGE, las velocidades de datos que se alcanzan en la práctica son menores que las teóricas, reportándose Qi et al. (2003) para la modificación A, tasas de $650 \mathrm{Kbps}$ en ambientes urbanos, $570 \mathrm{Kbps}$ en suburbanos y 500 Kbps en zonas rurales.

El SITAMM es adaptable a la plataforma inalámbrica disponible, ya sea EDGE, Ev-DO o cualquier otra tecnología de comunicaciones emergente, como HSDPA (High Speed Downlink Packet Access) o WiMAX (Worldwide Interoperability for Microwave Access). En las pruebas del SITAMM realizadas con EDGE, se mantuvo una tasa promedio de $48 \mathrm{~Kb}$ ps para la videoconferencia, $\mathrm{y}$ se observaron degradaciones y retardos indeseables en la señal de video. Con Ev-DO, en su versión original, se mejoró el desempeño del SITAMM, ya que se mantuvo una tasa promedio cercana a los $250 \mathrm{Kbps}$ a una velocidad máxima de $100 \mathrm{Km} / \mathrm{h}$. Con el uso de la tecnología 3G disponible en México, el SITAMM permitió mantener una sesión de videoconferencia a $250 \mathrm{kbps}$ (lo cual es aceptable desde el punto de vista técnico) con transmisión simultánea de signos vitales, a una velocidad máxima de $100 \mathrm{~km} / \mathrm{hr}$. Un aspecto importante a tomar en cuenta es la asimetría del canal inalámbrico, ya que esta condición impacta en la calidad de la videoconferencia que se transmite de la UME al SC-SITAMM, ya que este segmento del canal es de menor capacidad que el del SC-SITAMM a la UME. El contar con redes inalámbricas robustas, eficientes y de mejores capacidades de transmisión (4G ó WiMax) incrementará el desempeño del sistema.

\section{b) Aspectos de normatividad}

Un aspecto importante a considerar cuando se desea implantar un sistema de teleasistencia médica, es la integración de este sistema con la infraestructura de tecnologías de la información (TI) de los hospitales y es en este punto donde los estándares y la normatividad juegan un papel importante. Sin embargo, en México, al igual que en muchos países, la regulación en el área de la telemedicina es incipiente, y en la mayoría de los casos, es prácticamente nula. Por esta razón, es de especial interés realizar un estudio sobre los procesos involucrados en la teleasistencia médica, particularmente en casos de urgencias, e identificar aquellos que deben ser normados para garantizar la confidencialidad, seguridad e integridad de la información, así como su interoperabilidad con los sistemas hospitalarios.

Dado que la información que se transmite desde una unidad móvil o semimóvil puede viajar a través de redes públicas de telecomunicaciones como redes de 
3G, 4G o WiMax, es necesario definir los estándares de encriptación para garantizar la seguridad y anonimidad de la información. Este es un punto importante, ya que si de la información que se transmita desde la unidad móvil se tomarán decisiones sobre el tipo de cuidado o tratamiento que se le debe dar al paciente mientras es trasladado al hospital, se debe garantizar que la información no sea manipulada por un agente externo. Por otro lado, al tener la capacidad de acceder al expediente clínico electrónico del paciente en cuestión desde una unidad móvil, es necesario proteger la información para cumplir con lo establecido en la norma oficial mexicana NOMSSA-068, la cual especifica quiénes tienen acceso a la información contenida en el expediente clínico.

Otro punto interesante es la interacción que puede existir entre el sistema de teleasistencia médica y el expediente clínico electrónico. El sistema de teleasistencia médica debe tener la capacidad de saber qué buscar, dónde buscar y cómo procesar la información del expediente clínico electrónico del paciente. Esta información debe estar almacenada de la misma manera en los diferentes hospitales, de lo contrario, si cada hospital almacena de manera diferente la información, los beneficios de este sistema se verían limitados al sistema hospitalario para el que fue diseñado.

Así mismo, es necesario establecer cómo la información que se recaba en la unidad móvil, por ejemplo, los signos vitales, pueden ser almacenados en el expediente clínico electrónico para que puedan ser usados como una referencia posteriormente.

Los beneficios del uso de las tecnologías de la información en el sector salud radican en la interoperabilidad de los sistemas. La movilidad actual de la población a nivel local y nacional, implica cambios en el uso de los sistemas de salud, por ejemplo, entre diferentes clínicas del IMSS, del ISSSTE o del sistema privado. Esta condición hace más compleja la implantación de un expediente clínico electrónico único a nivel nacional, ya que se involucran aspectos técnicos, demográficos, socioeconómicos, jurídicos y políticos.

\section{Conclusiones}

La implantación de un sistema de teleasistencia en movimiento para aplicaciones en entornos hospitalarios presenta retos tecnológicos importantes, sin embargo, existen también retos operativos, regulatorios, normativos y de adopción tecnológica (aceptación del uso de la tecnología por parte de los usuarios), que deben ser considerados para el aprovechamiento de las bondades del sistema desarrollado. Uno de los aspectos más im- portantes a considerar es la integración del sistema de emergencias en movimiento a la infraestructura informática del hospital, tanto al expediente clínico electrónico, como a los sistemas de despliegue de información, pizarrones electrónicos o computadoras portátiles, que permita a los tomadores de decisión dictaminar de forma adecuada, precisa y expedita sobre las acciones que el paramédico debe ejecutar en el transcurso del lugar de la emergencia hacia el hospital.

Otro aspecto crucial es la definición de procesos y normas del uso de la tecnología disponible dentro del entorno de urgencias del hospital en forma estandarizada. Los aspectos de adopción son de particular importancia y juegan un papel crucial en el aprovechamiento integral de las capacidades de los sistemas de teleasistencia móvil desarrollados. Ya ha sido documentada (Agrawal et al., 2007) la importancia de los procesos regulatorios y normativos en entornos hospitalarios.

Este es un campo abierto con gran potencial de investigación, desarrollo e innovación, ya que se involucran aspectos de carácter multidisciplinario e interdisciplinario que deben ser incorporados a las tecnologías disponibles y por desarrollar.

Desde el punto de vista tecnológico, el transmitir señales de video bidireccional y signos vitales desde un vehículo en movimiento hacia un centro de atención de urgencias presenta retos importantes en cuanto a mantener conectividad durante todo el trayecto del vehículo y lograr resoluciones que permitan realizar diagnósticos adecuados, que en los casos de urgencias pueden ser la diferencia entre la vida o muerte del paciente.

Para cumplir con estos requerimientos, es importante seleccionar el proveedor de servicios inalámbricos que proporcione la mejor cobertura y capacidad de ancho de banda a precio competitivo. El SITAMM descrito en el presente artículo tiene un gran potencial de aplicación no sólo en entornos hospitalarios, sino también en otras áreas como son protección civil y transportes o traslados especializados. Estas aplicaciones podrán detonar el desarrollo de sistemas más eficientes en cuanto se tenga acceso a tecnologías inalámbricas emergentes de mayores capacidades tecnológicas como son 4G y WiMax móvil.

En suma, los retos más importantes de cualquier desarrollo de TIC aplicado al sector salud es lograr su integración con los sistemas informáticos ya existentes o por ponerse en operación, así como la interoperabilidad de equipos y sistemas en forma segura, privada y eficiente. Es por ello que los aspectos de normatividad y adopción tecnológica son cruciales para la implantación de dichos desarrollos. 


\section{Referencias}

Agrawal R., Grandison T., Johnson C., Kiernan J. Enabling the 21st Century Health Care Information Technology Revolution. Communications of the ACM, 50(2):35-42. 2007.

Bennett S. McRobb S., Farmer R. Object-Oriented Systems Analysis and Design Using UML, McGraw-Hill, USA, 2001, pp. 239-244.

Cypher D., Chevrollier N., Montavont N., Golmie N. Prevailing Over Wires in Healthcare Envirenments: Benefits and Challenges. IEEE Communications, 4(4):56-63. 2006.

Ericsson [en línea]. The evolution of EDGE. White Paper, 2007 [fecha de consulta 15 de enero 2010]. Disponible en: http://www.ericsson.com/news/090901_evolution_of_edge_20100510174715

Gómez-González A. Programa Nacional de Telesalud [en línea]. 2001 [fecha de consulta 15 de enero 2010]. Disponible en: http://ciberhabitat.gob.mx/hospital/telesalud/

Hospitales Digitales. Suplemento especial: Política Digital, Nexos [en línea]. 2007, Núm. 35, pp 39-40. [fecha de consulta 15 de enero 2010] Disponible en: http://www.politicadigital.com. mx/pics/edito/multimedia/476/num_35_multimedia.pdf
Istepanian R.S.H., Jovanov E., Zhang Y.T. M-health: Beyond Seamless Mobility for Global Wireless Healthcare Connectivity. IEEE Transactions Information Technology in Biomedicine, 8(4):405-414. 2004.

Li B., Qin Y., Low C.P., Gwee C.L. A Survey on Mobile WiMAX [Wireless BroadBand Access]. IEEE Communications Magazine, 45(12):70-75. 2007.

Poon C.C.Y., Yuan-Ting Z. A Novel Biometrics Method to Secure Wireless Body Area Sensor Networks for Telemedicine and M-Health. IEEE Communications, 4(4):73-81. 2006.

Qi-Bi R., Brown R., Cui Dongzhe, Gandhi A.D., Ching-Yao H., Vitebsky S. Performance of 1xEV-DO Third-Generation Wireless High-Speed Data Systems. Bell Labs Technical Journal, 7(3):97-107. 2003.

Rappaport T.S. Wireless Communications Principles and Practice, $2^{\underline{a}}$ ed., New Jersey, Prentice-Hall PTR, 2002, pp. 117-248.

RFC 3261[en línea]. SIP: Session Initiation Protocol. Internet Official Protocol Standards. 2002, pp. 8-55. [fecha de consulta 15 de enero 2010]. Disponible en: http://www.tekelec.com/ SIPReferenceGuide/rfc/?refID $=5$

\section{Semblanza de los autores}

Raúl Rivera-Rodríguez. Titulado como maestro en ciencias en electrónica y telecomunicaciones por parte del Centro de Investigación Científica y de Educación Superior de Ensenada (CICESE) en 1997. Realizó el trabajo de tesis en 1996, con duración de un año, en la Escuela Nacional Superior de Telecomunicaciones (ENST), París, Francia, trabajando en el área de gestión de redes de alta velocidad. Se tituló por el Instituto Tecnológico de Sonora (ITSON) en la carrera de ingeniero en electrónica en 1994. Actualmente realiza estudios de doctorado en la Facultad de Ingeniería-Mexicali de la Universidad Autónoma de Baja California (UABC) en temas de servicios de comunicación inalámbrica aplicados a la teleasistencia médica en movimiento.

Arturo Serrano-Santoyo. Obtuvo el grado de doctor en ciencias en ingeniería eléctrica en 1980 por el Centro de Investigación y Estudios Avanzados (CINVESTAV). Ha sido consultor para la Organización de Estados Americanos y la Organización de las Naciones Unidas en el área de sistemas y redes de comunicaciones. Desde 1993, es miembro de número de la Academia Mexicana de Ingeniería. Es además perito en telecomunicaciones en México y actualmente funge como director de innovación y desarrollo del Centro de Investigación Científica y Educación Superior de Ensenada (CICESE) y como miembro del Sistema Nacional de Investigadores Nivel III.

Raúl Tamayo-Fernández. Se tituló como ingeniero en electrónica en 1995 por la Facultad de Ingeniería de la Universidad Autónoma de Baja California (UABC), obteniendo el Premio al Mérito Escolar por sus estudios. En 1997, se tituló como maestro en ciencias en electrónica y telecomunicaciones en el Centro de Investigación Científica y de Educación Superior de Ensenada (CICESE). Actualmente realiza sus estudios de doctorado en la $\mathrm{UABC}$, en el área de las comunicaciones inalámbricas emergentes.

Álvaro Armenta-Ramade. Realizó sus estudios de ingeniería en electrónica en la Facultad de IngenieríaEnsenada de la Universidad Autónoma de Baja California (UABC). En el 2001, obtuvo el grado de maestro en ciencias en telecomunicaciones por el Centro de Investigación Científica y de Educación Superior de Ensenada (CICESE) con el tema de calidad de servicio en redes basadas en IP. Actualmente realiza los estudios de doctorado en la UABC, en el área de normatividad en la telesalud. 